\title{
THE OCCURRENCE OF ANOMALOUS WINDS AND THEIR SIGNIFICANCE
}

\author{
M. A. ALAKA \\ National Hurricane Research Project, U.S. Weather Bureau, Miami, Fla. \\ [Manuscript received May 9, 1961; revised August 14, 1961]
}

\begin{abstract}
Observational evidence is provided for the occurrence of anomalous winds which represent an anticyclonic rotation in space, and a mechanism for their development is suggested. The unstable nature of these winds and the role they play in the development of certain types of atmospheric disturbances is then discussed, and it is suggested that anomalous winds provide the dynamic mechanism for triggering hurricane formation and for the observed deepening of troughs downstream from intense pressure ridges. Finally it is noted that although the observational evidence presented is for the occurrence of anomalous winds over small regions of the atmosphere, their development is dependent on large-scale processes and their effect extends beyond the area where they occur.
\end{abstract}

\section{INTRODUCTION}

The gradient wind equation is a quadratic and thus has two solutions. One of these solutions, appropriate to anticyclonic flow, represents a clockwise rotation in space and is, therefore, in the opposite sense to the earth's rotation. Meteorologists have traditionally given little attention to this solution and some even consider it as an algebraic accident with little or no physical significance. Although there are cogent reasons to believe that winds corresponding to this solution do not occur on a large scale, the contention, ipso facto, that this solution is of no importance is not justified and has probably had far-reaching effects in eliminating from consideration some promising a venues of research in connection with the development of atmospheric disturbances.

In recent years there has been a slow trickle of evidence, both theoretical and observational, that these so called "anomalous winds" occur more frequently than had been suspected. But, by and large, meteorologists are still not sufficiently aware of these winds nor of their importance. The purpose of the present study is not only to provide further evidence of the occurrence of anomalous winds, but also to demonstrate that these winds are germane to the development of dynamic instability in curved airflow and, as such, are relevant to the development of certain types of atmospheric circulations.

\section{ELEMENTARY DYNAMICS OF ANTICYCLONIC MOTION}

To introduce the problem, we shall review some elementary aspects of the dynamics of anticyclonic motion.

The gradient wind equation may be written:

$$
K_{t} V^{2}+f V=b_{n}
$$

were $K_{t}=1 / R_{t}=$ trajectory curvature considered positive for cyclonic motion; $V=$ magnitude of the horizontal wind; $f=$ Coriolis parameter; and $b_{n}=-\alpha \partial p / \partial n=$ pressure gradient force. From equation (1)

$$
V=-\frac{f}{2 K_{t}}\left(1 \pm \sqrt{1+\frac{4 K_{t} b_{n}}{f^{2}}}\right)
$$

Since $V$ must always be positive, the solution with the plus sign has a physical meaning only when $K_{t}$ is negative; i.e., when the flow is anticyclonic.

We shall confine our discussion to anticyclonic flow and put $K_{t}^{\prime}=-K_{t}$. The two solutions of equation (2) reduce to:

$$
V_{1}=\frac{f}{2 K_{t}^{\prime}}\left(1-\sqrt{1-\frac{4 K_{t}^{\prime} b_{n}}{f^{2}}}\right)
$$

and

$$
V_{2}=\frac{f}{2 K_{t}^{\prime}}\left(1+\sqrt{1-\frac{4 K_{t}^{\prime} b_{n}}{f^{2}}}\right)
$$

From the above equations it can be seen that

$$
V_{1} \leq \frac{f}{2 K_{t}^{\prime}}
$$

and

$$
V_{2} \geq \frac{f}{2 K_{\ell}^{\prime}}
$$

We differentiate equation (1) with respect to $V$

$$
\frac{d b_{n}}{d V}=2 V K_{t}+f
$$

and note that $d b_{n} / d V$ can be zero; i.e., $b_{n}$ has an extreme value if $K_{t}$ is negative. This value is reached when 


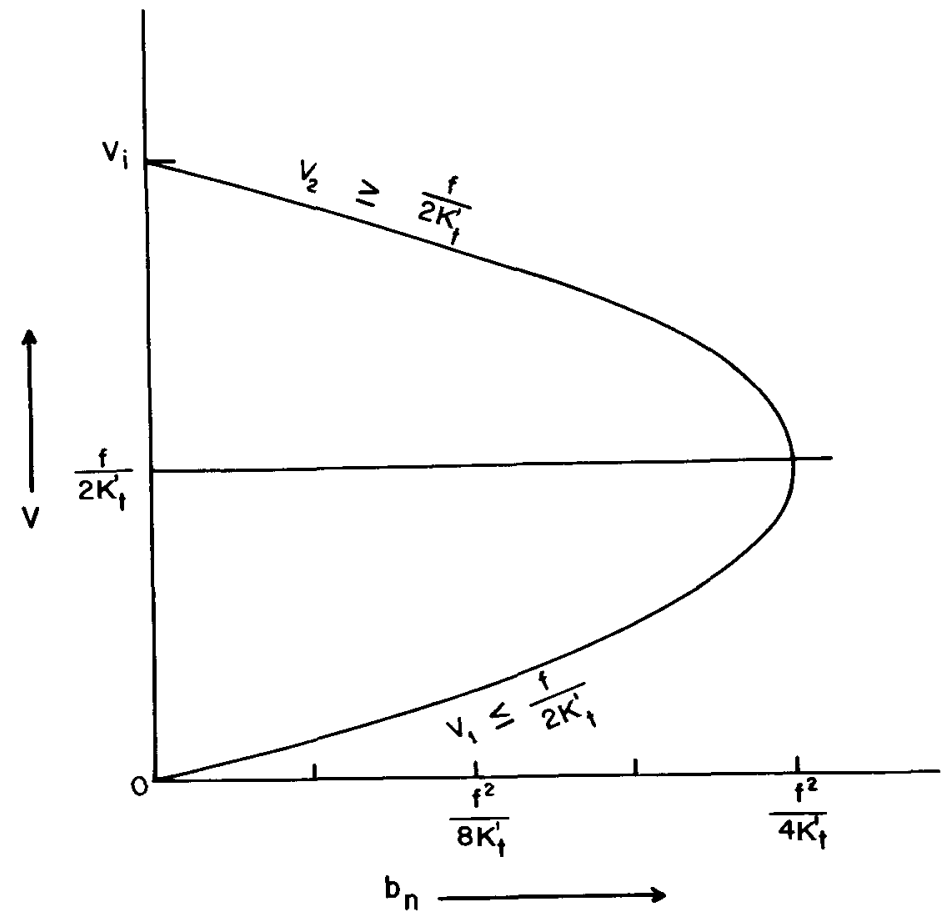

FIgURE 1.-Variation of gradient anticyclonic wind speed with pressure gradient.

$$
V=-\frac{f}{2 K_{t}}=\frac{f}{2 K_{t}^{\prime}}
$$

and represents the maximum value

$$
b_{n}=-\frac{f^{2}}{4 K_{\imath}}=\frac{f^{2}}{4 K_{t}^{\prime}}
$$

which the pressure gradient can attain in anticyclonic motion.

Figure 1 shows the variation of $V$ with $b_{n}$ in accordance with equation (1). It is seen that the two solutions meet at $V=f / 2 K_{t}^{\prime}$ when the pressure gradient is at its maximum value $f^{2} / 4 K_{i}^{\prime}$. From this point, where the wind is equal to twice the geostrophic wind $V_{g}$, the two solut ons $V_{1}$ and $V_{2}$, corresponding respectively to equations (3) and (4) branch out. $\quad V_{1}$ decreases with decreasing pressure gradient and vanishes when the latter becomes zero. This corresponds to the conditions normally observed in the atmosphere. We shall therefore term $V_{1}$ the normal solution of the anticyclonic gradient wind equation. $V_{2}$, on the other hand, increases with decreasing pressure gradient and at $b_{n}=0$ reaches the value $V_{i}=f / K_{i}^{\prime}$ and the flow becomes inertial. Following Gustafson [7], we shall term $V_{2}$ the anomalous solution.

Figure 2 shows the variation of the gradient anticyclonic wind with trajectory curvature, assuming a constant pressure gradient. It is seen that, in contrast with

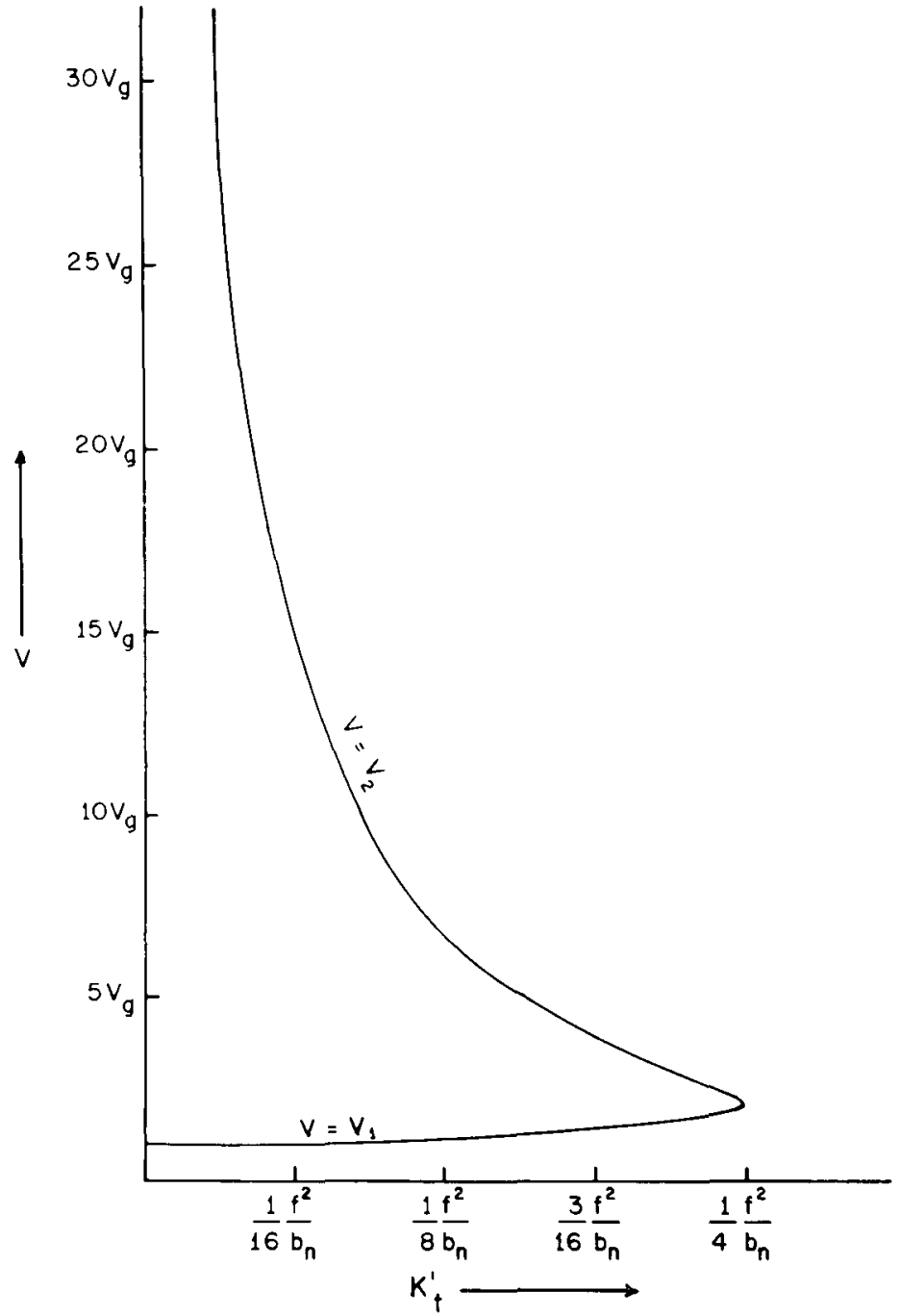

FIGURE 2.-Variation of gradient anticyclonic wind speed with trajectory curvature. $V_{g}$ denotes the geostrophic wind.

normal anticyclonic winds which increase with increasing curvature, anomalous winds show a sharp decrease with increasing curvature.

As mentioned in the introduction, meteorologists in general have attributed little importance to the anomalous solution on the basis that it is seldom, if ever, realized in the atmosphere [5] or that its occurrence is limited to smallscale mechanically produced vortices, or to atmospheric eddies produced by friction [11]. The standard arguments in support of this view are usually some variation of the following:

a. The anomalous solution requires a clockwise rotation in space and, therefore, in the opposite sense to that of the earth. There is no known mechanism capable of producing such a motion on a large scale.

b. By expanding the quantity under the radical sign in equations (3) and (4), we obtain 


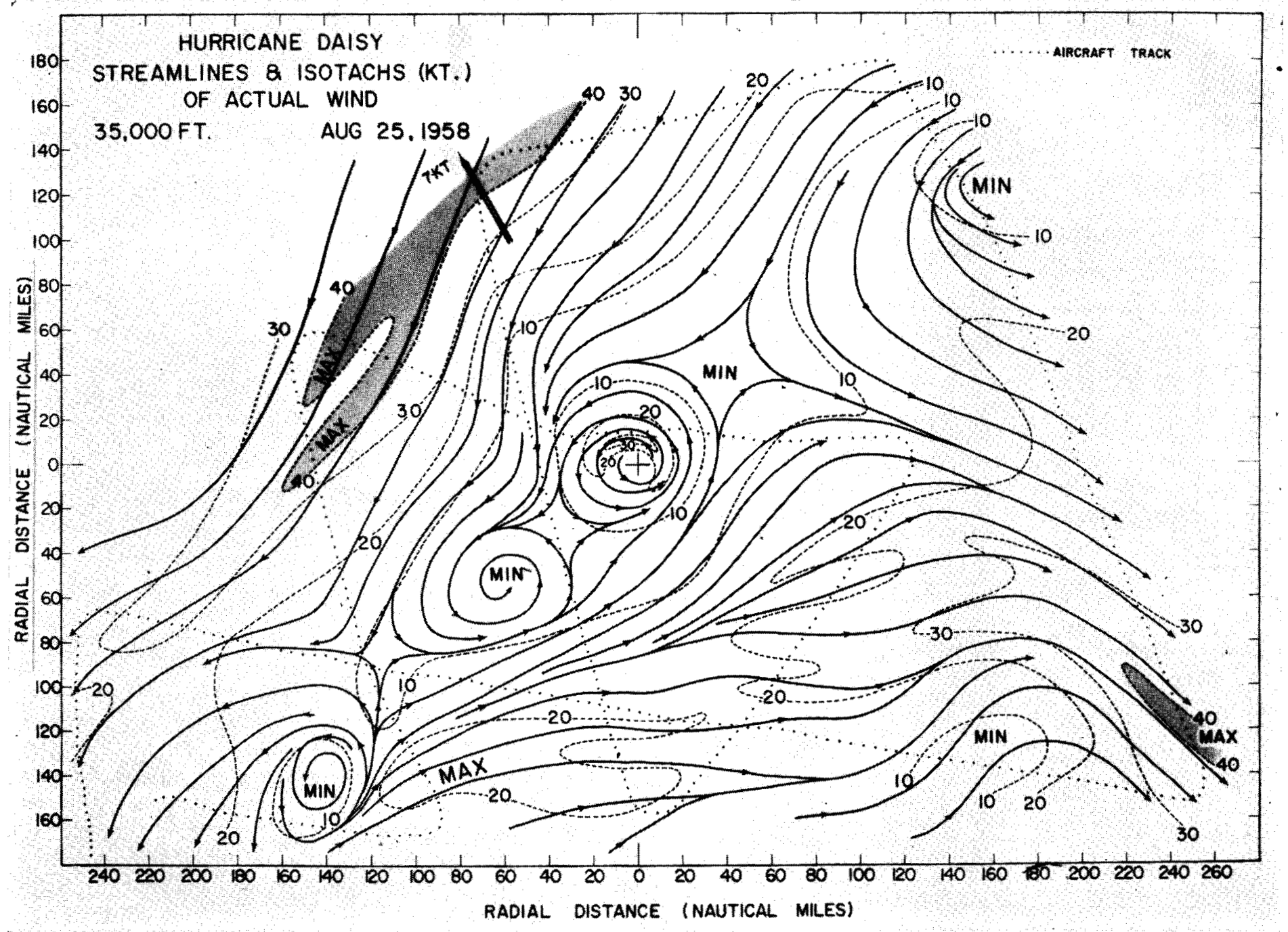

Fig URE 3.--Wind field at 35,000 ft. (pressure altitude, U.S. Standard), around hurricane Daisy on August $25,1958$.

$$
\begin{aligned}
V_{1} & =\frac{f}{2 \bar{K}_{t}^{\prime}}\left[1-\left(1-\frac{2 K_{t}^{\prime} b_{n}}{f^{2}}-\frac{2 K_{t}^{\prime 2} b_{n}{ }^{2}}{f^{4}}+\cdots\right)\right] \\
& =\frac{b_{n}}{f}+\frac{K_{t}^{\prime} b_{n}{ }^{2}}{f^{3}}+\cdots
\end{aligned}
$$

and

$$
\begin{aligned}
V_{2} & =\frac{f}{2 K_{t}^{\prime}}\left[1+\left(1-\frac{2 K_{t}^{\prime} b_{n}}{f^{2}}-\frac{2 K_{t}^{\prime 2} b_{n}{ }^{2}}{f^{4}}+\cdots\right)\right] \\
& =\frac{f}{K_{t}^{\prime}}-\frac{b_{n}}{f}-\frac{K_{t}^{\prime} b_{n}{ }^{2}}{f^{3}}+\cdots
\end{aligned}
$$

$V_{1}$ is thus continuous when the curvature decreases indefinitely and the isobars are nearly straight. On the other hand, for indefinitely small curvature, $V_{2}$ becomes infinitely large and would require an infinite supply of energy, which is not available in the atmosphere [5].

\section{EVIDENCE OF THE OCCURRENCE OF ANOMALOUS WINDS}

In contrast with the above arguments, there are others which indicate that both solutions for gradient anticyclonic flow are satisfied. Godson [6], for instance, considers a moving anticyclonic streamline system and expresses the gradient wind relation as follows:

$$
V=\frac{f R_{c}+C \cos \psi}{2} \pm\left[\left(\frac{f R_{c}+C \cos \psi}{2}\right)^{2}-f R_{c} V_{g}\right]^{1 / 2}
$$

where $R_{c}=$ radius of contour curvature, $C=$ speed of streamline system, $\psi=$ angle from the direction of motion of the streamline system to the wind direction. Godson argues that if $C \cos \psi<0$, it is possible to obtain two values of $V$ both of which are greater than $2 V_{\mathbf{g}}$ and thus correspond to the classically rejected root. On the other 


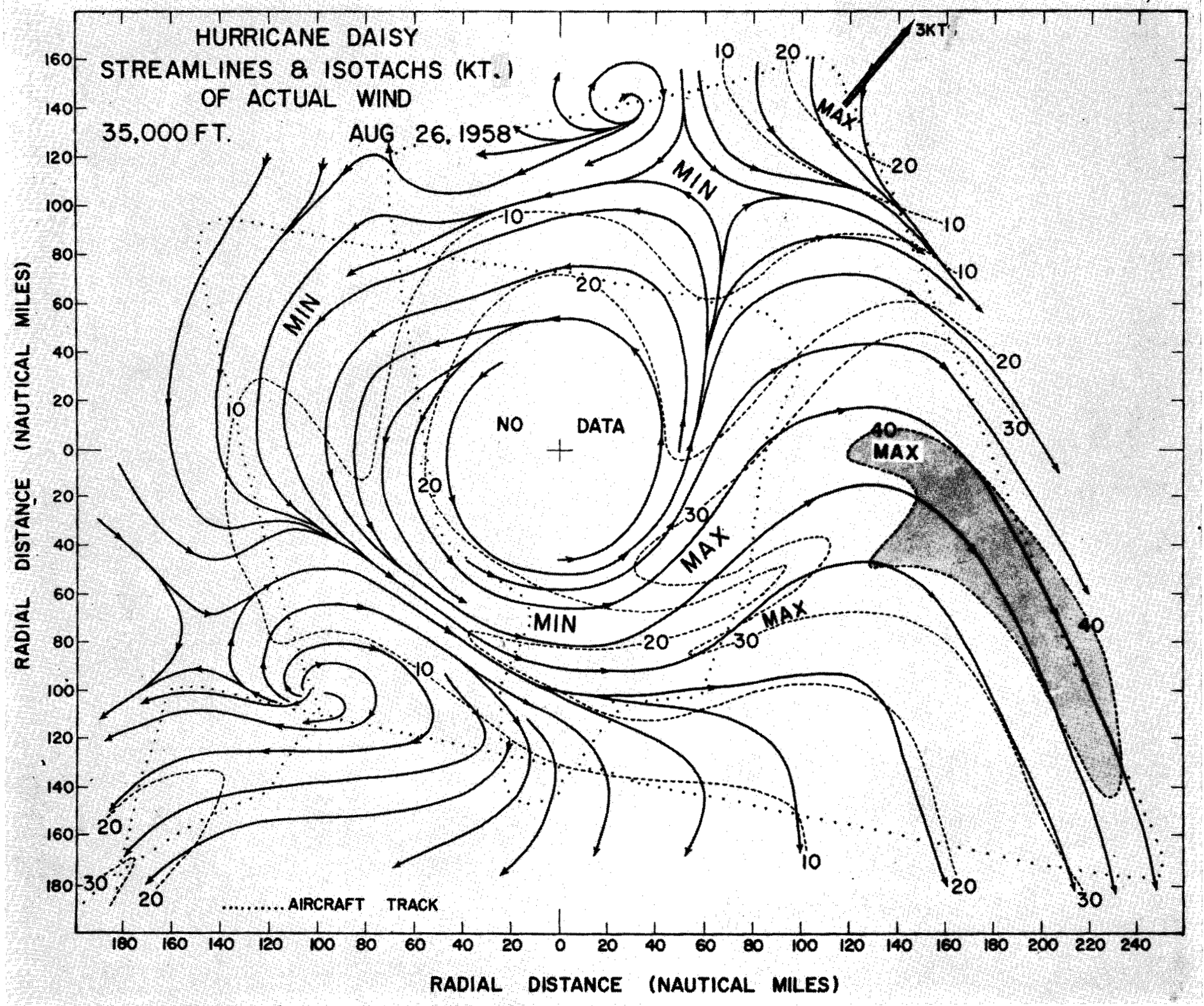

Figure 4.-Wind field at 35,000 ft. around hurricane Daisy on August 26, 1958.

hand if $C \cos \psi>0$, it is possible to obtain values of $V$ both of which are less than $2 V_{g}$ and thus correspend to the classically accepted root. He concludes that both roots of the gradient wind equation for anticyclonic flow occur in the atmosphere.

On the observational side, indirect evidence that anomalous winds occur was provided by Gustafson [7] who compared observed winds with geostrophic winds in a moving pressure ridge at $700 \mathrm{mb}$. and applied the following criteria which follow directly from equations (5) and (6) above:

and

$$
\left.\begin{array}{l}
V_{1} \leq 2 V_{g} \cos \beta \\
V_{2} \geq 2 V_{g} \cos \beta
\end{array}\right\}
$$

where $\beta$ is the deviation in direction between the observed and geostrophic winds, measured clockwise from due east. In a 3-day sequence Gustafson found consistent positive deviation from geostrophic directions to the west of the moving ridge ard similarly consistent negative deviations to the east of it.

More recently, Angell [3] has furnished more direct evidence of the occurrence of anomalous flow from an evaluation of transosonde flights at $300 \mathrm{mb}$. In one instance the transosonde performed a full clockwise loop in 36 hours with an estimated angular velocity of $-0.48 \times$ $10^{-4}$ sec. $^{-1}$ This represents anomalous flow since the angular velocity of the earth at that locality $\left(30^{\circ} \mathrm{N}\right.$.) is $0.36 \times 10^{-4}$ sec. $^{-1}$ More significantly, perhaps, the transosonde trajectories suggested the occasional occur- 


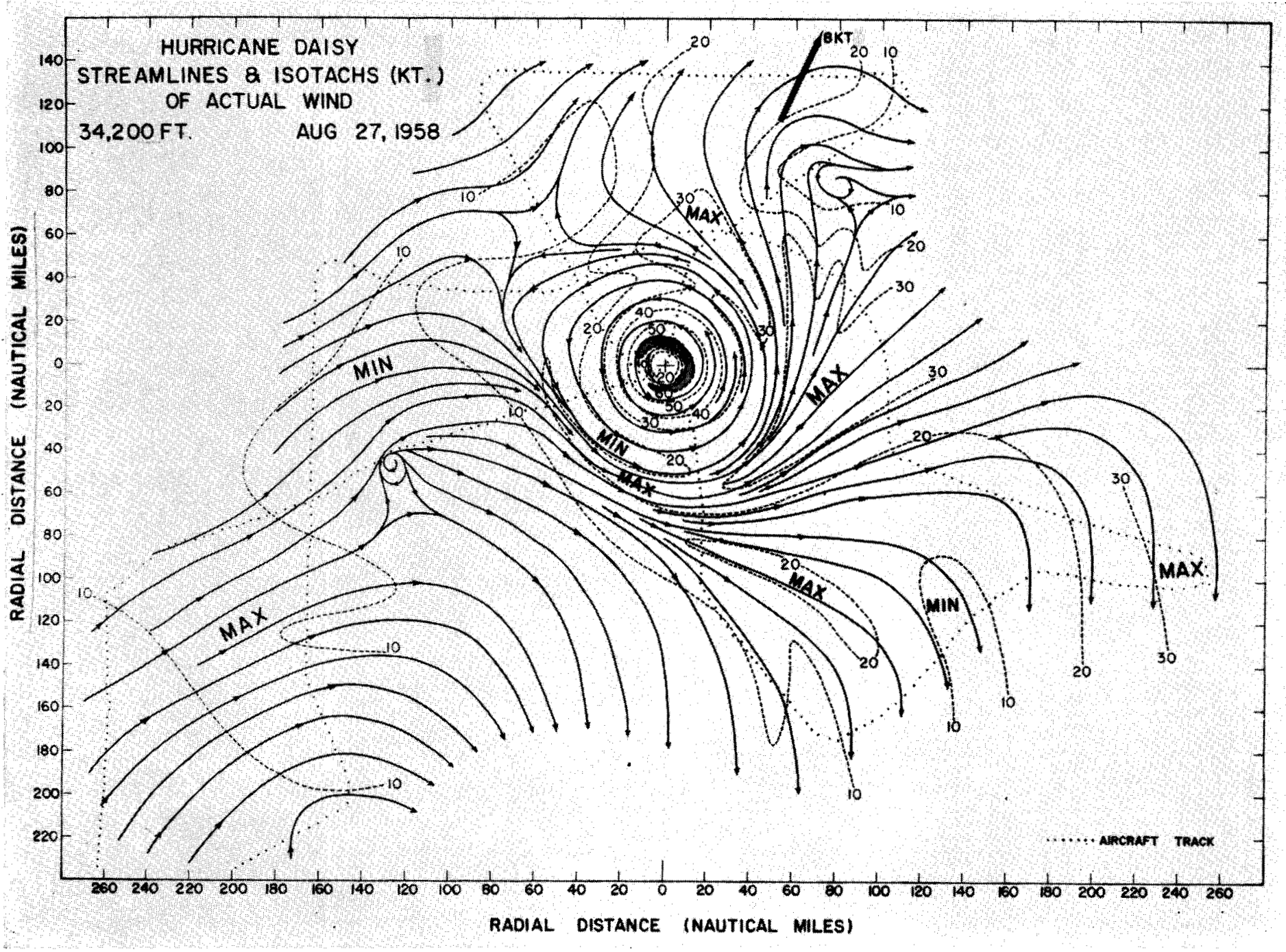

FigURE 5.-Wind field at 34,200 ft. around hurrieane Daisy on August 27, 1958.

rence of anomalous flow on the ridges of long waves in the westerlies. The significance of this fact will be discussed in section 7 , below.

Since the summer of 1956 , the National Hurricane Research Project (NHRP), U.S. Weather Bureau, has been operating three specially instrumented airplanes to make detailed observations in and near hurricane cores. A discussion of the characteristics and properties of the instrumentation was given by Hilleary and Christensen [10] and will not be repeated here. Among the most successful missions flown were those in connection with hurricane Daisy which developed near the Bahamas on August 24, 1958. This hurricane had a well-defined and concentrated wind circulation and presented a clear-cut radar configuration which greatly facilitated the location of the storm core. Flight missions were made at different levels on four days from August 25 to August 28, 1958. Among the elements measured were the wind, the temperature, and the radio and pressure altitudes. Quasiinstantaneous values of these parameters were punched on cards at specified intervals ranging from 10 seconds away from the core, to 2 seconds in the core. The punched cards were then evaluated by machine processing and the various parameters were plotted on a coordinate system fixed with respect to the storm center.

Figures 3, 4, and 5 show the wind field obtained by analyzing the observations made in the upper troposphere on August 25, 26, and 27. In making these analyses, it was necessary to regard as quasi-simultaneous, observations which, in fact, were made over a period of several hours. It is, however, believed that this shortcoming does not invalidate the results obtained.

From the wind fields thus obtained, values of the speed and direction were plotted on a rectangular grid of points 20 n.mi. apart. From these, computations of the quantity $2 V K_{t}$ were made on the IBM 650 computer. The curvature of the trajectory $K_{t}$ was computed from the following relations:

If $u$ and $v$ are the westerly and southerly components of the wind, and if we define 


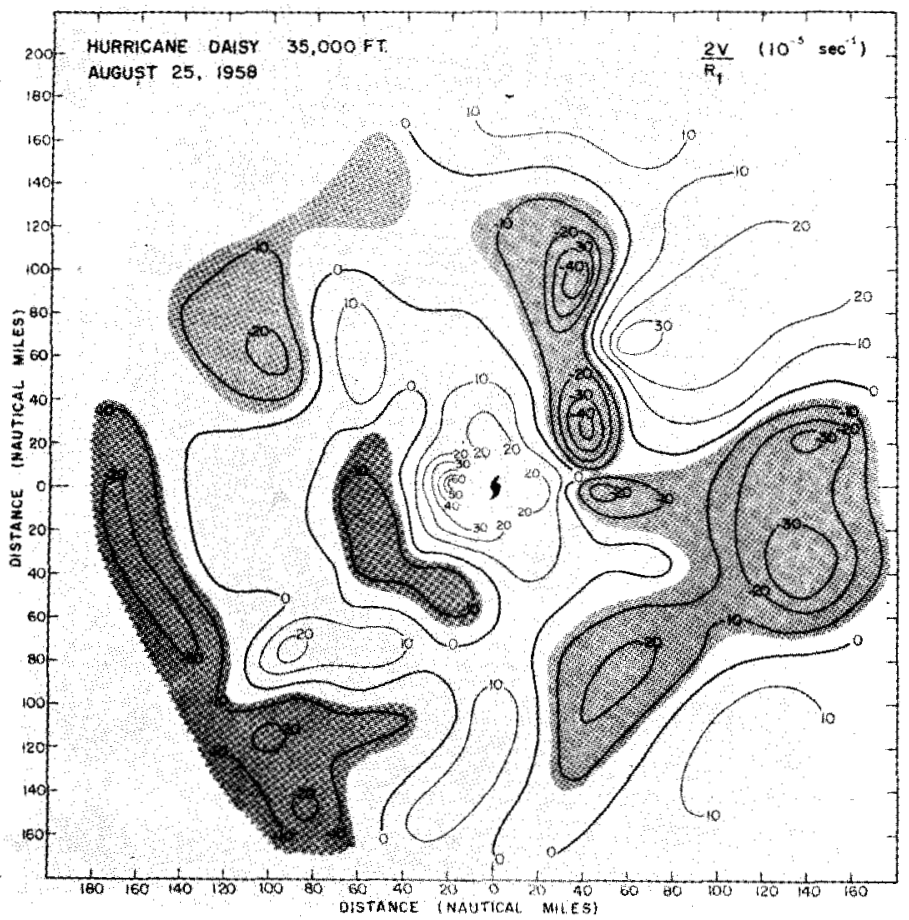

Figure 6.-Field of the quantity $2 V / R_{t}$ at 35,000 feet (pressure altitude, U.S. Standard), around hurricane Daisy on August 25, 1958. Areas with anomalous winds are shaded.

$$
\psi=\tan ^{-1} \frac{u}{v}
$$

then

$$
\begin{aligned}
K_{t} & =-\frac{d \psi}{d s}=\cos ^{2} \psi\left(\frac{u}{v^{2}} \frac{d v}{d s}-\frac{1}{v} \frac{d u}{d s}\right) \\
& =\frac{1}{V^{2}}\left(u \frac{d v}{d s}-v \frac{d u}{d s}\right) \\
& =\frac{1}{V^{3}}\left(u \frac{d v}{d t}-v \frac{d u}{d t}\right)
\end{aligned}
$$

If the motion of the storm is represented by the vector $c$ with components $c_{x}$ and $c_{y}$ in the east and north dircetions, respectively, and if we assume steady state conditions,

$$
\begin{aligned}
K_{t}= & \frac{1}{V^{3}}[u(\mathbf{V}-\mathbf{c}) \cdot \nabla v-v(\mathbf{V}-\mathbf{c}) \cdot \nabla u] \\
= & \frac{1}{V^{3}}\left\{u\left[\left(u-c_{x}\right)\right] \frac{\partial v}{\partial x}+\left(v-c_{y}\right) \frac{\partial v}{\partial y}\right. \\
& \left.-v\left[\left(u-c_{x}\right) \frac{\partial u}{\partial x}+\left(v-c_{y}\right) \frac{\partial u}{\partial y}\right]\right\}
\end{aligned}
$$

Figures 6,7 , and 8 represent fields of the quantity $2 K_{t}$ $V=2 V / R_{t}$ corresponding to the wind fields of figures 3 , 4 , and 5, respectively. Regions where this quantity is negative and numerically greater than the Coriolis parameter, i.e., areas where the winds are anomalous, are shaded. The consistency of the patterns of these areas

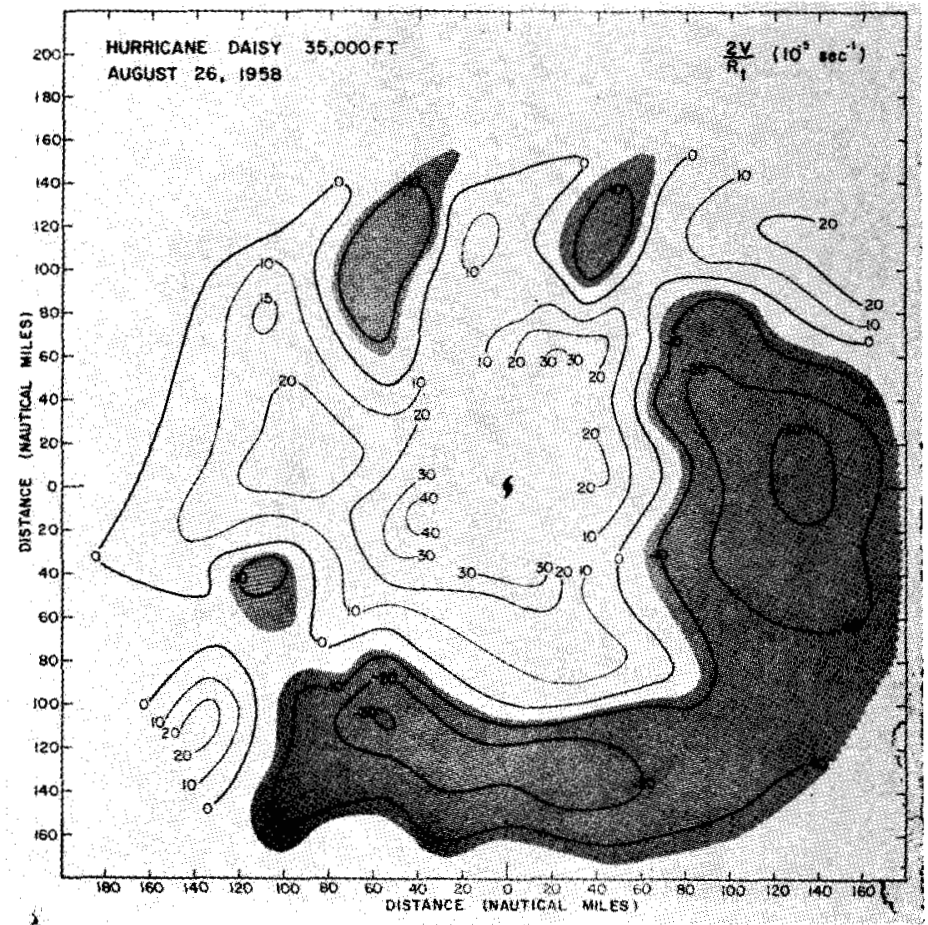

Figure 7.-Field of the quantity $2 V / R_{t}$ at $35,000 \mathrm{ft}$. for August 26, 1958 .

from day to day attests to their authenticity and demonstrates beyond any reasonable doubt that anomalous winds do occur. It will be noted, however, that the areas covered by these winds represent narrow strips of the order of 1 to 2 degrees of latitude in width, and are, therefore, likely to escape detection by ordinary synoptic analysis. In addition, the vertical extent of these winds is also limited, as can be noted from the fact that they are not found in figure 9 which represents the field of $2 V / R_{t}$ a few thousand feet below that of figure 6 . This is perhaps the reason why meteorologists in general have failed to realize both the reality and importance of these winds. The latter stems from the fact that they represent an important mechanism for the development of atmospheric instability as we shall now proceed to show.

\section{THE CONCEPT OF INSTABILITY OF ATMOSPHERIC MOTION}

The concept of instability in the atmosphere appeared with the initial developments in the theory of atmospheric disturbances. As early as 1878, Rayleigh [13] investigated conditions under which small displacements at the boundary between two air streams grow into larger disturbances. At about the same time, Helmholtz [8] found that, under certain conditions, unstable gravity waves developed at the interface between two liquids of different density and velocity. The above studies culminated in the polar front theory of cyclone formation. 


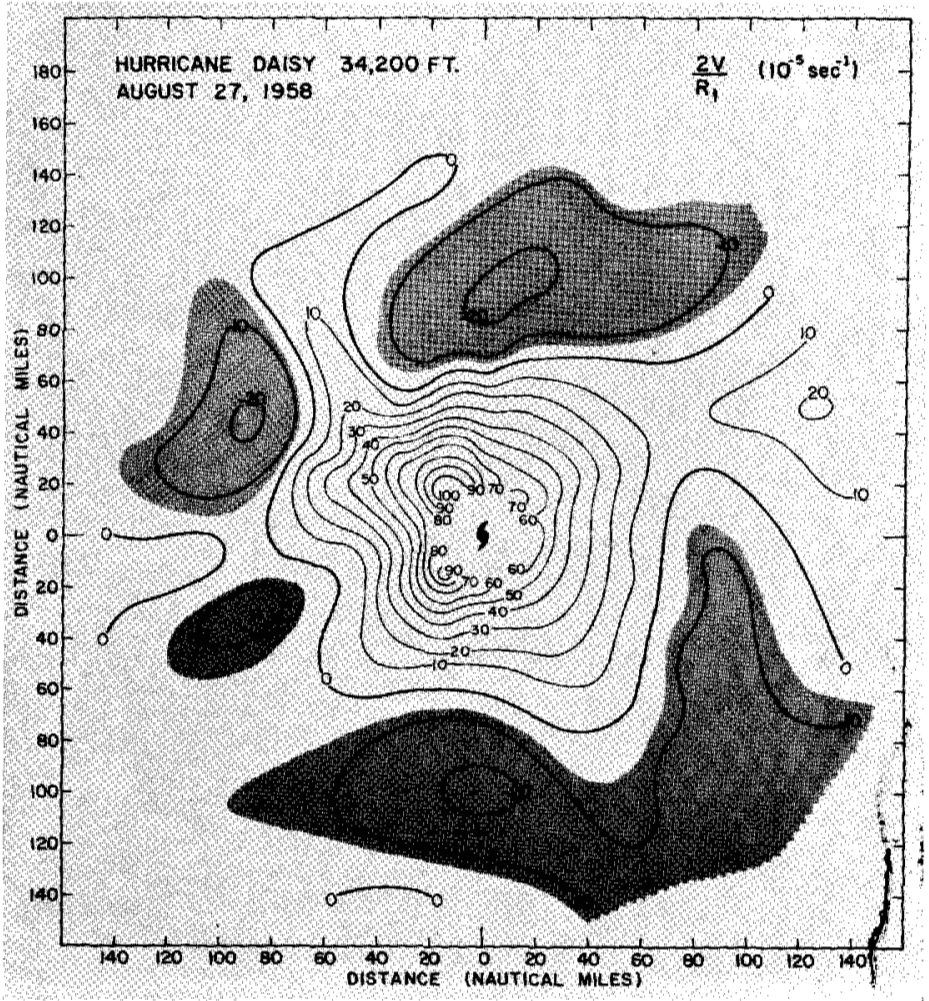

FIGURE 8.-Field of the quantity $2 V / R_{t}$ for August 27, 1958, at $34,200 \mathrm{ft}$.

Paralleling the above studies, another series of investigations, beginning with those of Helmholtz [9] and Rayleigh [14] attempted to link instability in air currents with the quasi-horizontal distribution of the kinematic properties of the currents. This type of instability is usually known as dynamic instability.

In recent years, attention has been redirected to still another type of instability known as baroclinic instability. This type of instability, the importance of which was first pointed out by Margules [12], occurs by virtue of the vertical wind shear.

There is little doubt that the above three types of instability all play an important role in the atmosphere and there is an obvious need for a unified theory which combines them and brings out their relative importance and their interrelations. In the absence of such a theory, we must consider the particular type of instability which appears to have the most direct bearing on the problem at hand. In the present case, the importance of anomalous winds is best demonstrated by studying the circumstances attending the development of the so-called dynamic instability, with especial reference to atmospheric currents in curved motion.

\section{DYNAMIC INSTABILITY IN CURVED AIRFLOW}

In 1936, Solberg [16] investigated the conditions under which dynamic instability occurs in a steady symmetrical polar vortex which is initially in gradient equilibrium.

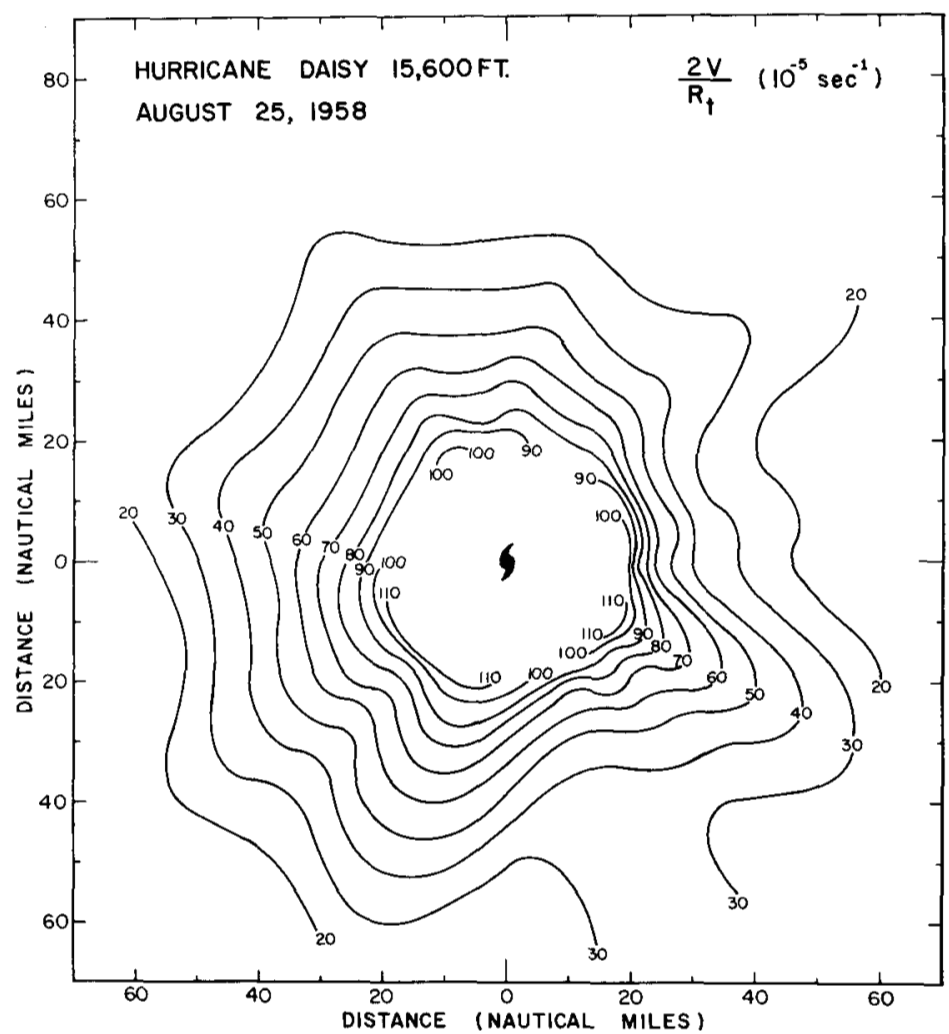

Figure 9.-Field of the quantity $2 V / R_{t}$ at $15,600 \mathrm{ft}$. for August 25, 1958.

Solberg's approach may be extended to any steady vortex in which the tangential variations of the wind are negligible in comparison with those in the radial direction.

I tet us then consider such a vortex in which the angular speed $\omega$ is a function of the distance $R$ from the axis of rotation. We shall proceed by the standard method and impose a small perturbation on this steady vortex and find out the circumstances under which the frequency of the total perturbed motion becomes imaginary.

If we disregard friction, we can express the equilibrium of forces acting on the steady vortex by the following equation,

$$
\frac{V^{2}}{R^{2}} \mathbf{R}+\frac{2 \Omega V}{R} \mathbf{R}-\theta \nabla \pi+\mathbf{g}=0
$$

where $V$ is the magnitude of the speed, $\Omega$ the earth's rotation, $\theta$ the potential temperature, $\mathbf{s}$ the gravity vector, and $\pi=c_{p}(p / 1,000)^{R / c_{p}}$.

Let us now apply a uniform radial impluse to all the particles at distance $R^{\prime}$ from the axis so that at the time $t=t$ the particles form another circle with radius $R$. We stipulate that at any instant, the perturbed particles immediately acquire a pressure equal to that of the points which they are occupying at that instant. We further assume that the perturbed particles, in their displacement over a small distance $S$ from their equilibrium position conserve both their potential temperature $\theta^{\prime}$ and their 
absolute angular momentum $M_{a}^{\prime}=V^{\prime} R+\Omega R^{\prime 2}$ so that

and

$$
\left.\begin{array}{c}
\theta-\theta^{\prime}=\mathbf{S} \cdot \nabla \theta \\
M_{a}-M_{a}^{\prime}=\mathbf{S} \cdot \nabla M_{a}
\end{array}\right\}
$$

In addition to the forces indicated in equation (17), the perturbed particles sustain an acceleration $d^{2} \mathbf{S} / d t^{2}$ so that their motion is determined by the equation.

$$
\frac{d^{2} \mathbf{S}}{d t^{2}}+\frac{V^{\prime 2}}{R^{2}} \mathbf{R}+\frac{2 \Omega V^{\prime}}{R} \mathbf{R}-\theta^{\prime} \nabla \pi+\mathbf{g}=0
$$

From equations (17) and (19), we obtain by subtraction

$$
\frac{d^{2} \mathbf{S}}{d t^{2}}-\frac{V^{2}-V^{\prime 2}}{R^{2}} \mathbf{R}-\frac{2 \Omega}{R}\left(V-V^{\prime}\right) \mathbf{R}+\left(3-\theta^{\prime}\right) \nabla \pi=0
$$

Now

$$
V^{2}-V^{\prime 2}=\left(V-V^{\prime}\right) \cdot 2 V-(\delta V)^{2}
$$

where $\delta V=V-V^{\prime}$ is a perturbation quantity, the square of which may be neglected. Equation (20) thus becomes

$$
\frac{d^{2} \mathbf{S}}{d t^{2}}-\left[\frac{2}{R}(\omega+\Omega)\left(V-V^{\prime}\right)\right] \mathbf{R}+\left(g-\theta^{\prime}\right) \nabla \pi=0
$$

We now express the above equation in terms of the conservative quantity $M_{a}$ and make use of the relations in. (18); we obtain

$$
\begin{aligned}
\frac{d^{2} \mathbf{S}}{d t^{2}}-\left[\frac{2}{R^{2}}(\omega+\Omega)\left(\mathbf{S} \cdot \nabla M_{a}\right)+\frac{2}{R}\left(\omega^{2}+3 \omega \Omega\right.\right. & \left.\left.+2 \Omega^{2}\right) \delta R\right] \mathbf{R} \\
+(\mathbf{S} \cdot \nabla \theta) \nabla \pi & =0
\end{aligned}
$$

If the perturbation is very small compared to the dimensions of the vortex, i.e., if $\left(R-R^{\prime}\right) / R \approx 10^{-1}$, the term in $\delta R$ in the above equation may be neglected and we finally have

$$
\frac{d^{2} \mathbf{S}}{d t^{2}}-\left[\frac{2}{R^{2}}(\omega+\Omega)\left(\mathbf{S} \cdot \nabla M_{a}\right)\right] \mathbf{R}+(\mathbf{S} \cdot \nabla 6) \nabla \pi=0
$$

Let us assume that the displacement $\mathbf{S}$ is proportional to a function of the form $e^{i \nu t}$ and project equation (24) along two orthogonal directions: one corresponding to the vertical and the other to a radial direction. If the components of $\mathbf{S}$ along these directions are given by $z$ and $r$, respectively, the projections of equation (24) along these directions are:

and

$$
\left.\begin{array}{l}
\nu^{2} z-\frac{2 \omega_{a r}}{R}\left(r \frac{\partial M_{a}}{\partial r}+z \frac{\partial M_{a}}{\partial z}\right)+\frac{\partial \pi}{\partial z}\left(r \frac{\partial \theta}{\partial r}+z \frac{\partial \theta}{\partial z}\right)=0 \\
\nu^{2} r-\frac{2 \omega_{a z}}{R}\left(r \frac{\partial M_{a}}{\partial r}+z \frac{\partial M_{a}}{\partial z}\right)+\frac{\partial \pi}{\partial r}\left(r \frac{\partial \theta}{\partial r}+z \frac{\partial \theta}{\partial z}\right)=0
\end{array}\right\}
$$

where $\omega_{a}$ denotes the absolute rotation $(\omega+\Omega)$.

For nontrivial values of $r$ and $z$, the determinant of the above system of linear equations vanishes and we obtain

$$
\begin{aligned}
\nu^{4}+\nu^{2} & {\left[\left(\frac{\partial \pi}{\partial z} \frac{\partial \theta}{\partial z}+\frac{\partial \pi}{\partial r} \frac{\partial g}{\partial r}\right)+\left(\frac{2 \omega_{a z}}{R} \frac{\partial M_{a}}{\partial r}-\frac{2 \omega_{a r}}{R} \frac{\partial M_{a}}{\partial z}\right)\right] } \\
& -\left(\frac{2 \omega_{a z}}{R} \frac{\partial \pi}{\partial z}+\frac{2 \omega_{a r}}{R} \frac{\partial \pi}{\partial r}\right)\left(\frac{\partial M_{a}}{\partial z} \frac{\partial \theta}{\partial r}-\frac{\partial M_{a}}{\partial r} \frac{\partial \theta}{\partial z}\right)=0
\end{aligned}
$$

Examining the order of magnitude of the various terms in equation (26), we find that

$$
\begin{aligned}
& \frac{\partial \pi}{\partial z} \frac{\partial \theta}{\partial z}+\frac{\partial \pi}{\partial r} \frac{\partial \theta}{\partial r}=\nabla \pi \cdot \nabla \theta \approx 10^{-4} \\
& \frac{2}{R}\left(\omega_{a z} \frac{\partial M_{a}}{\partial r}-\omega_{a r} \frac{\partial M_{a}}{\partial z}\right)=-2 \omega \cdot \nabla \times \mathrm{V}_{a} \approx 10^{-8} \\
& \frac{2}{R}\left(\omega_{a z} \frac{\partial \pi}{\partial z}+\omega_{a r} \frac{\partial \pi}{\partial r}\right)\left(\frac{\partial M_{a}}{\partial z} \frac{\partial \theta}{\partial r}-\frac{\partial M_{a}}{\partial r} \frac{\partial \theta}{\partial z}\right) \\
& \left.=\left(2 \omega_{a} \cdot \nabla \pi\right)\left(\nabla \theta \cdot \nabla \times \mathbf{V}_{a}\right) \approx 10^{-12}\right)
\end{aligned}
$$

Solving for $\nu^{2}$ and neglecting the last two terms of (27) in comparison with the first, we have

$$
\nu_{1}^{2}=-\nabla \pi \cdot \nabla \theta
$$

The value of this root is determined mainly by the static stability

$$
-\frac{\partial \pi}{\partial z} \frac{\partial \theta}{\partial z}=\frac{g}{\theta} \frac{\partial \theta}{\partial z}
$$

The second root $\nu_{2}{ }^{2}$ is obtained by dividing the third term of (27) by $\nu_{1}{ }^{2}$. Thus,

$$
\nu_{2}{ }^{2}=\frac{\frac{2}{R}\left(\omega_{a z} \frac{\partial \pi}{\partial z}+\omega_{a r} \frac{\partial \pi}{\partial r}\right)\left(\frac{\partial M_{a}}{\partial z} \frac{\partial \theta}{\partial r}-\frac{\partial M_{a}}{\partial r} \frac{\partial \theta}{\partial z}\right)}{\frac{\partial \pi}{\partial z} \frac{\partial \theta}{\partial z}+\frac{\partial \pi}{\partial r} \frac{\partial \theta}{\partial r}}
$$

The above equation will be greatly simplified if it is projected on an isentropic surface so that $\partial \theta / \partial r$ vanishes. In addition, since the angle between isobaric and isentropic surfaces is generally small, $(\partial \pi / \partial r) \approx 0$ and

$$
\nu_{2}^{2}=\left[2 \omega_{a z}(\nabla \times V)_{z}\right]_{\theta}
$$

Equation (31) is identical with Solberg's results for the polar vortex and states that the square of the frequency of the second root is equal to twice the product of the absolute rotation of the vortex and the vertical component of the absolute vorticity, as measured on an isentropic surface. If we now use a relative frame of reference fixed with respect to the earth, we have

$$
\nu_{2}^{2}=\left[\left(\frac{2 V}{R_{t}}+f\right)(\zeta+f)\right]_{0}
$$

Since we are considering a steady vortex, the streamlines coincide with the trajectory and $R=R_{t}$. The frequency $\nu_{2}$ becomes imaginary and the motion unstable when

$$
\left[\left(\frac{2 V}{R_{t}}+f\right)(\zeta+f)\right]_{\theta}<0
$$




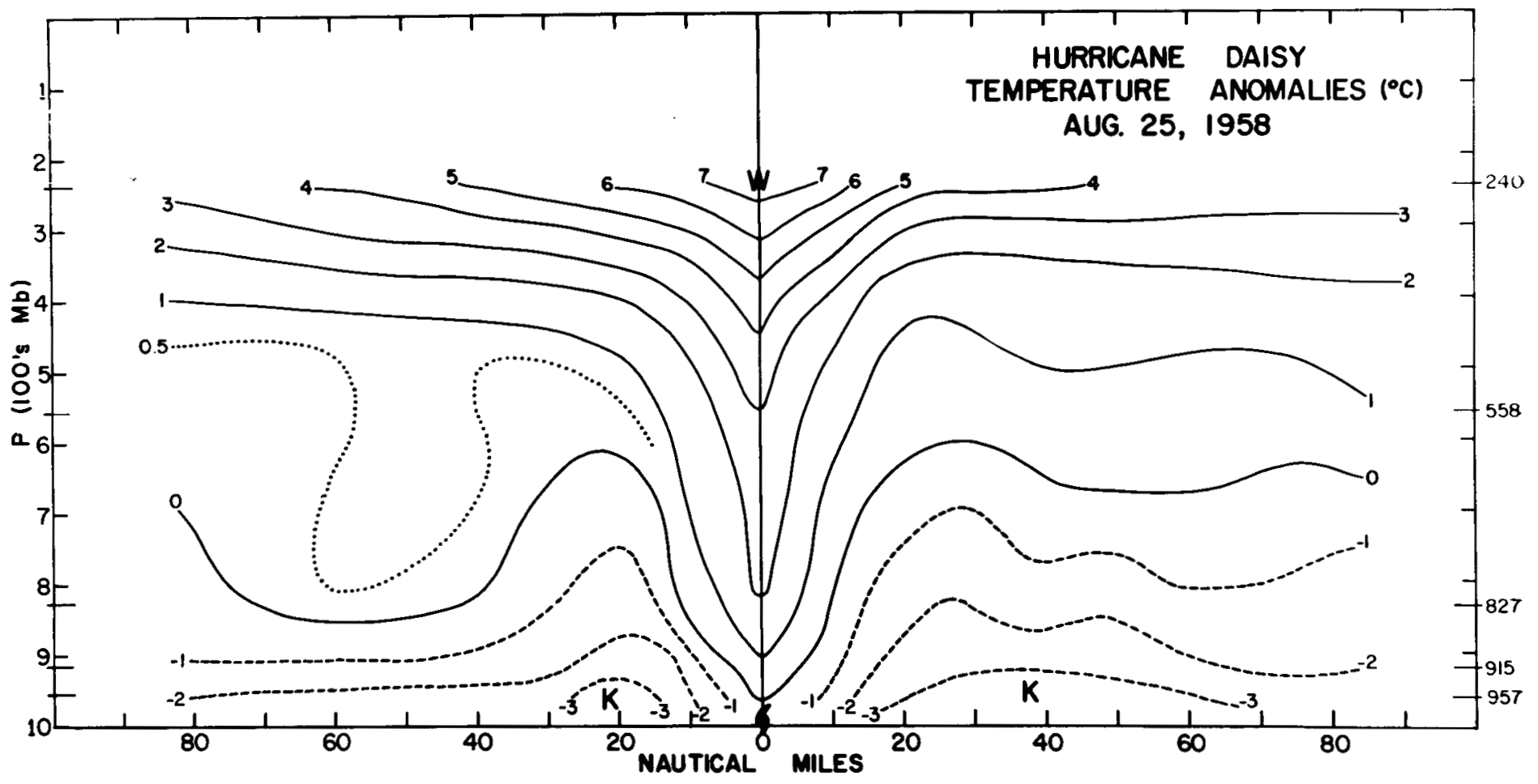

FIGURE 10.-Vertical cross-section of temperature anomalies from the mean August atmosphere in a direction perpendicular to that of the motion of hurricane Daisy, August 25, 1958. Pressures shown at the right edge indicate the levels at which data were available (analysis by J. A. Colón).

In other words, dynamic instability occurs with normal winds provided the absolute vorticity, as measured on an isentropic surface, is negative. Alternatively, instability occurs with positive absolute vorticity provided the wind, as measured on an isentropic surface, is anomalous.

The above criterion is identical with that obtained by van Mieghem [17]. The latter, however, did not envisage the possibility of the occurrence of anomalous winds and, therefore, equated the condition for the release of dynamic instability with the occurrence of negative absolute vorticity.

It should be noted that, according to the theory of equations, the second root may be obtained by subtracting $\nu_{1}^{2}$ from the negative value of the coefficient of $\nu^{2}$ in equation (26). If we then neglect the vertical wind shear $\left(\partial M_{a} / \partial z=0\right)$, we have

$$
\nu_{2}^{\prime 2}=\left(\frac{2 V}{h_{t}}+f\right)(\zeta+f)
$$

which is similar to equation (32) except that the quantities are now measured on a level surface. Equation (34) is identical with the criterion for dynamic instability obtained by Sawyer [15].

Physically, the operation of the above criterion may be visualized by studying the balance of forces on an anticyclonic vortex which is initially in gradient balance. Let a particle be given an impulse toward lower pressure.
If the flow is unstable, the particle tends to continue in the direction of the impulse. There are two cases to consider:

Case I: Instability with Normal Winds. -If the winds are normal, there is instability if the pressure gradient along the path of the particle increases at such a rate that the speed of the particle remains subgradient. This would require a rapid outward increase in anticyclonic rotation or a rapid decrease in cyclonic rotation expressed by the inequality

$$
\left[\frac{1}{R} \frac{\partial}{\partial r}(V R)\right]_{\theta}<-f
$$

Case II: Instability with Anomalous Winds.-From figures 1 and 2 , it is scen that if the wind regime is anomalous, the speed increases with decreasing pressure gradient and trajectory curvature. Therefore, if the trajectory curvature of the particle remains constant, the pressure gradient along the trajectory should decrease at a fast rate; otherwise, the speed of the perturbed particle will eventually reach a speed greater than that appropriate for gradient equilibrium and its acceleration will be checked. Actually, however, the trajectory curvature decreases as the particle moves toward lower pressure. Therefore, instability can occur with a slower rate of pressure gradient decrease or, if the curvature decreases rapidly, instability can occur even with a slow outward increase of the pressure gradient. 


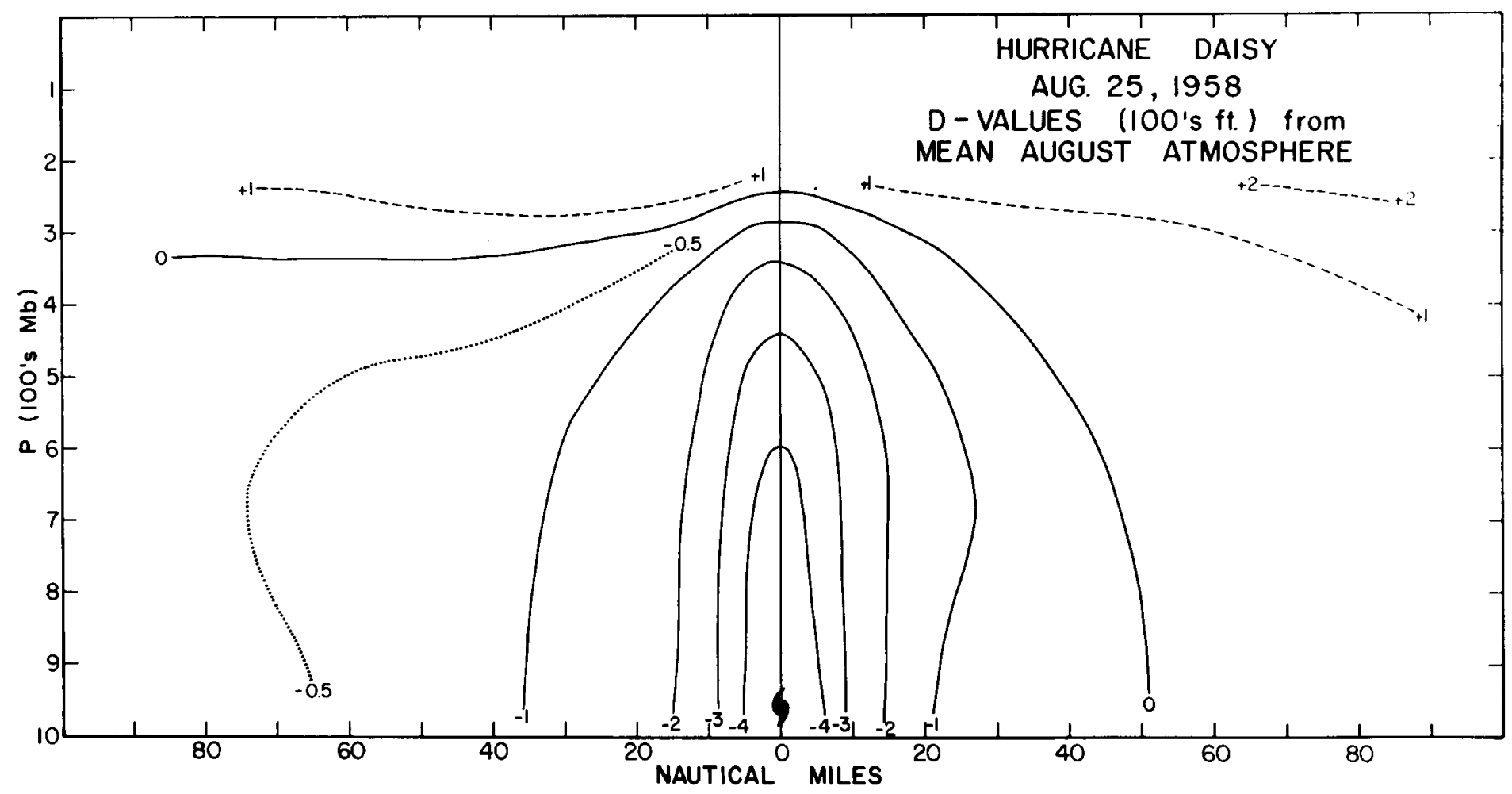

FIgURE 11.-Vertical cross-section of deviations of the altitudes of pressure surfaces from the mean August atmosphere (" $D$ " values) in a direction perpendicular to that of hurricane Daisy, August 25, 1958 (analysis by J. A. Colón).

Equation (33) states that the motion is unstable so long as the combined effect of the change of wind speed curvature is such that

$$
\left[\frac{1}{R} \frac{\partial}{\partial r}(V R)\right]_{\theta}>-f
$$

\section{A MECHANISM FOR THE DEVELOPMENT OF ANOMALOUS WINDS}

An insight into the manner in which anomalous winds develop may be gained by considering the circulation theorem of Bjerknes which may be written [11]:

$$
\left.\begin{array}{rl}
\frac{d C_{a}}{d t} & =\frac{d C}{d t}+2 \Omega \frac{d \sum}{d t} \\
& =N_{\alpha_{1}-p}
\end{array}\right\}
$$

$C_{a}=\oint_{Q} \mathrm{~V}_{u} \cdot \delta \mathbf{r}=$ absolute circulation around a closed curve $Q$.

$C=\oint_{Q} \vee \cdot \delta \mathrm{r}=$ relative circulation around the same curve.

$\sum=$ the equatorial projection of the area within the curve $Q$.

$N_{\alpha_{1}-p}=$ the number of pressure-volume solenoids enclosed by the curve $Q$.
A comparison of figures 6,10 , and 11 indicates that there are few solenoids at the level and location where the anomalous winds occur. We may, therefore, set

$$
\frac{d C}{d t}+2 \Omega \frac{d \sum}{d t}=0
$$

or

$$
C+2 \Omega \sum=\text { constant }
$$

If we approximate the hurricane to a simple circular vortex, the circulation at a distance $R$ from the center is

$$
C=2 \pi R^{2} \omega
$$

and

$$
\sum=\pi R^{2} \sin \phi
$$

where $\phi$ is the latitude.

Substituting the above values of $C$ and $\Sigma$ in (39), we obtain

$$
R^{2}\left(\omega+\Omega_{z}\right)=\text { constant }
$$

whence it can be seen that, in a circular vortex, disregarding the solenoid term in equation (37) is equivalent to assuming conservation of absolute momentum - a concept which is known to be applicable to upper-level hurricane circulation.

According to equation (42), if the vortex has horizontal convergence, the circles contract and $\omega$ increases. If, on the other hand, the circles expand as a result of horizontal 
divergence, $\omega$ decreases. In this case, in order that $\omega$ should decrease sufficiently so that

$$
\left.\begin{array}{cc} 
& \omega+\Omega_{z} \rightarrow 0 \\
\text { i.e., } & \\
\frac{2 V}{R}+f \rightarrow 0
\end{array}\right\}
$$

$R$ must approach infinity. Since the expansion cannot proceed so far, we conclude that the anomalous winds in figures 4,5 , and 6 were not produced by a symmetrical horizontal divergence of the air particles which arrived at these higher levels by ascent in the core of the storm. Another mechanism must be sought to account for their occurrence.

From figure 1 it is clear that a change of regime from normal to anomalous winds would be more difficult to accomplish if the pressure gradient force is much lower than the maximum value $f^{2} / 4 K^{\prime}{ }_{t}$, since this would require a big jump in the wind speed. If, however, the pressure gradient is nearly equal to the maximum, a comparatively small impulse would be sufficient to shift an air particle from the lower to the upper branch of the $V$ curve of figure 1 .

Let us visualize initial conditions characterized by an anticyclonic air stream in gradient equilibrium. In figure 12 , let $A$ be the equilibrium position of a particle in this stream. Now let us visualize that the equilibrium is disturbed by an increase in the pressure gradient brought about, for instance, by an interaction between tropical and extratropical pressure systems. Two cases may be discussed: Case A.-The increase of the pressure gradient force is from $\mathrm{P}$ to $\mathrm{P}_{1}$, i.e., the resulting pressure gradient force is well below the maximum $f^{2} / 4 K_{t}^{\prime}$. 'The wind, having become subgradient, the particle turns toward lower pressure and accelerates. Having reached the equilibrium speed B, unless there is considerable damping, the particle may slightly overshoot this position to $B_{1}$. It then oscillates with decreasing amplitude about the equilibrium position until balance is finally reached at $B$.

Case B.-The increase of the pressure gradient force is from $\mathrm{P}$ to $\mathrm{P}_{2}$; i.e., the increased pressure gradient force is nearly equal to the maximum value $f^{2} / 4 K_{t}^{\prime}$. In this case, the speed of the accelerating particle, by overshooting the equilibrium position, may reach the value $\mathrm{B}_{3}$ which is in excess of the critical speed $f / 2 K_{t}^{\prime}$. Whereas at $B_{2}$ the speed of the particle is supergradient, and at $\mathrm{B}_{3}$ it is subgradient for the same pressure gradient. Thus, by overshooting the critical speed, the particle is henceforth constrained to move toward lower pressure and sustain further acceleration unless the distribution of the wind field along its path is such that the absolute vorticity is negative; in this case the motion will be stabilized in accordance with equation (33).

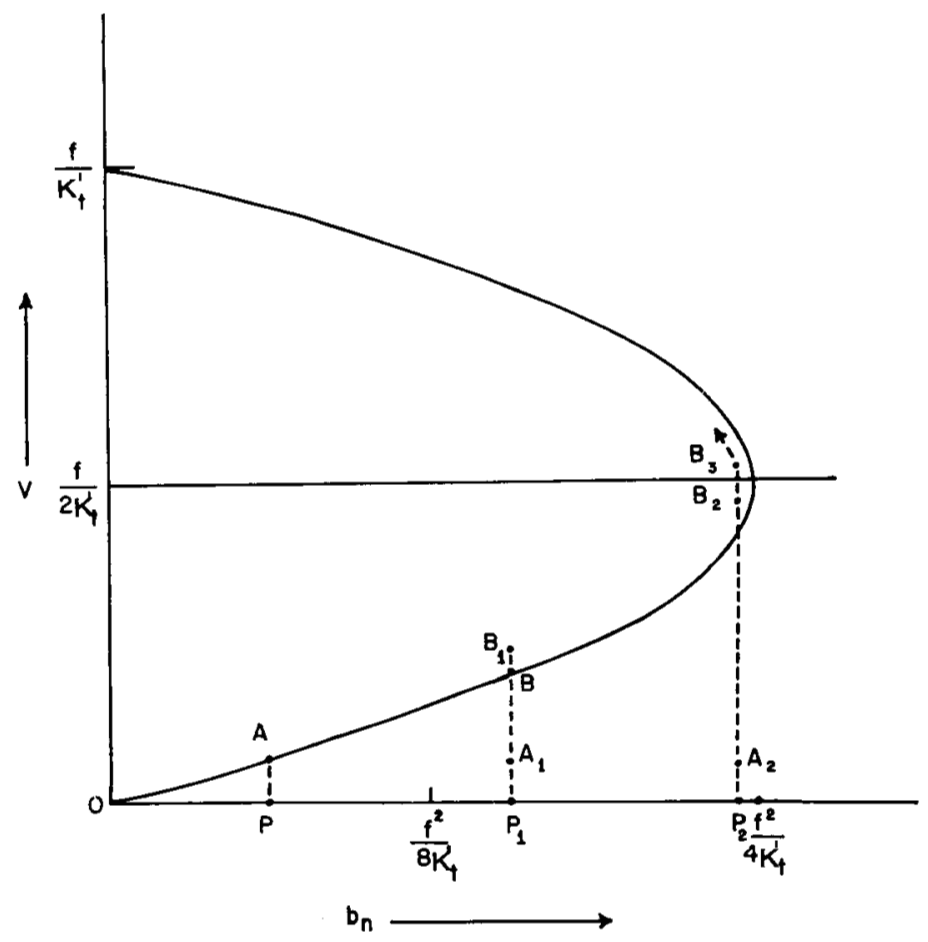

Figure 12.-Illustrating the development of anomalous winds (see text).

\section{EXAMPLES OF SITUATIONS IN WHICH ANOMALOUS WINDS PLAY A ROLE}

In a previous paper [1], the author suggested from theoretical considerations that the occurrence of anomalous winds in the manner described above provides a mechanism for triggering hurricane development. Details concerning how this is brought about and some observational evidence to this effect are given in a separate paper [2] and are beyond the scope of the present study. Here we would suggest that, in view of their essentially unstable nature, anomalous winds must play an important role not only in hurricane formation but also in other types of atmospheric development, especially those which occur downstream from a pressure ridge which has undergone marked intensification, or those accompanying the superposition of strong westerly winds on the crests of the long waves.

A case in point is that discussed by Bjerknes [4] linking the deepening of a wave trough with a strong intensification of the ridge upstream from it. Specifically, Bjerknes suggests that if the ridge intensifies so that the curvature of the contours exceeds a critical value

$$
\frac{f^{2}}{4 b_{n}}=\frac{f}{4 V_{g}}
$$

where $V_{g}$ denotes the geostrophic wind, the air particles cannot follow the contours and must perforce flow to- 


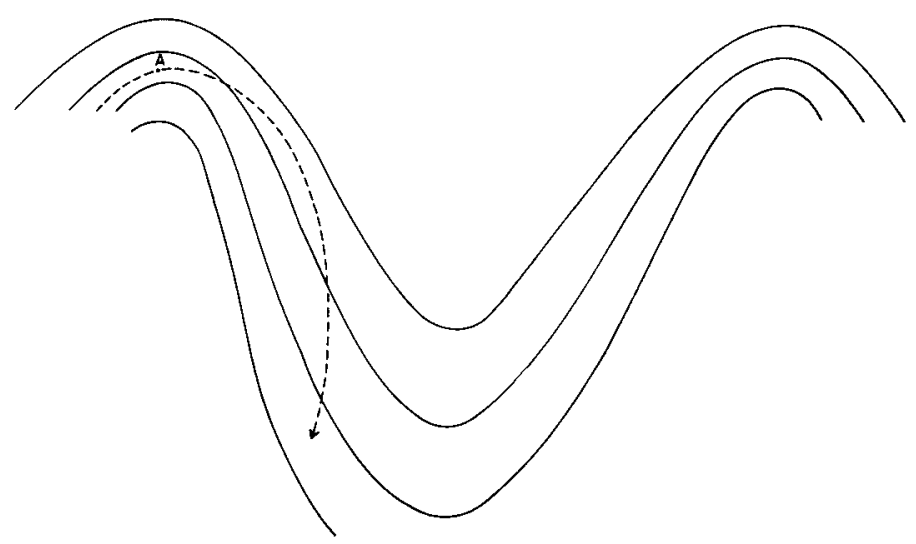

FIGURE 13.- Illustrating the cyclogenetic effect of anomalous winds. The dashed curve represents the probable trajectory of an air particle after its speed changes to the anomalous regime at the point $\mathrm{A}$.

ward lower pressure and accelerate. The acceleration results in supergradient winds which then curve back to higher pressure, the net result being a deepening of the trough downstream from the ridge.

Now that the likelihood of the occurrence of anomalous winds in situations analogous to that described above has been demonstrated, the deepening of the trough may be looked at in a new light. Jet us suppose that the wind becomes anomalous as a result of the strengthening of the pressure gradient to a value near the maximum possible for anticyclonic flow, and let us follow the trajectory of a particle situated at the point $\mathrm{A}$ in figure 13 , where the wind changes regime and becomes subgradient in the manner discussed in the previous section. As a result, the air particle moves to lower pressure and accelerates. In doing so, its curvature decreases, as can be seen from the figure. Unless the pressure gradient along the trajectory increases rapidly, the progressively decreasing curvature requires an increasingly stronger speed for balanced motion (see fig. 2) and the particle, therefore, continues to accelerate. However, if the curvature vanishes so that the particle follows a straight trajectory, the wind instantly becomes supergradient and the particle must curve back to higher pressure. In the mean, the particle must, therefore, follow a slightly anticyclonic trajectory and is constrained by the geometry of the pressure distribution to curve back to higher pressure, as shown by the dashed curve in figure 13 .

In this connection it is of interest to mention that Angell [3] has noted that anomalous flow toward higher pressure can occur continuously for several hours. Thus, by stipulating the occurrence of anomalous winds, we arrive at the same ultimate deepening envisaged by Bjerknes.

\section{CONCLUSION}

Although the observational evidence presented here is for the occurrence of anomalous winds over small regions of the atmosphere, it is significant to note:

A. that their occurrence may be dependent on largescale processes, such as those involving the intensification of pressure ridges or the latitudinal shift of the westerlies; and

B. that their effect extends beyond the area where they occur.

The fact that these winds can occur only in anticyclonic motion again demonstrates the vital regulatory role of the high pressure regions in determining the state and motion of the atmosphere.

\section{ACKNOWLEDGMENTS}

The author wishes to acknowledge his indebtedness to his colleague, Dr. Stanley L. Rosenthal, for his help in programming the computations mentioned in section 3 . The author also wishes to record his appreciation to Dr. José A. Colón for making available figures 10 and 11, and to Mr. Daryl T. Rubsam who is responsible for figures 3, 4, and 5. Finally, acknowledgment is due Mrs. Miree Moore and Mr. A. M. Recht for their help in plotting, and to Messrs. R. Carrodus and C. True for reproducing the figures.

\section{REFERENCES}

1. M. A. Alaka, "Dynamies of Upper-Air Outflow in Incipient Hurricanes," Geophysica, vol. 6, No. 3-4 1958, pp. 133-146.

2. M. A. Alaka, "The Occurrence of Dynamic Instability in Incipient and Developing Hurricanes." (To be published.)

3. J. K. Angell, "An Analysis of Operational 300-mb. Transosonde Flights from Japan in 1957-58," Journal of Metcorology, vol. 17, No. 2, Feb. 1960, pp. 20-35.

4. J. Bjerknes, "Extratropical Cyclones," Compendium of Meteorology, American Meteorological Society, Boston, 1951, pp. 577-598.

5. D. Brunt, Physical and Dynamical Meteorology, Cambridge University Press, 1939, $428 \mathrm{pp}$

6. W. L. Godson, "Some Remarks Concerning the Gradient Wind Equation," Bulletin of the American Meteorological Society, vol. 30 , No. 12, Decomber 1949, pp. 342-346.

7. A. F. Gustafson, "On Anomalous Winds in the Free Atmosphere," Bulletin of the American Meteorological Society, vol. 34, No. 5, May 1953, pp. 196-201.

8. H. Helmholtz, "Úber Atmosphärische Bewegungen," Meteorologische Zeitschrift, vol. 5, 1888, pp. 329 340 .

9. H. Helmholtz, "On Atmospheric Movements," Smithsonian Miscellaneous Collection, vol. 34, 1893, (translated by C. Abbe).

10. D. T. Hilleary and F. E. Christensen, "Instrumentation of National Hurricane Research Project Aircraft," National Hurricane Research Project Report No. 11, 1957.

11. J. Holmboe, G. E. Forsythe, and W. Gustin, Dynamic Meteorology, John Wiley and Sons, New York, 1954, 348 pp.

12. M. Margules, "Über die Energie der Stürme," Jahrbuch K. K., Zentralanstalt für Meteorologie und Geodynamik, Wien, Bd. XL, 1905, pp. 1-26.

13. Lord Rayleigh, "On the Instability of Jets," Proceedings of the Mathematical Society of London, vol. 10, 1878, pp. 4-13. 
14. Lord Rayleigh, "On the Stability of the Laminar Motion of an Inviscid Fluid," Philosophical Magazine, vol. 23, 1913, pp. $1001-1010$.

15. J. S. Sawyer, "Notes on the Theory of Tropical Cyclones," Ouarterly Journal of the Royal Meteorological Society, vol. 73, 1947, pp. 101-126.
16. H. Solberg, "Le mouvement d'inertie de l'atmosphère stable et son rôle dans la théorie des cyclones," Procès-verbaux, l'Association Météorologique, UGGI, Edinbourg 1936, Part II, 1939 , pp. 66-82.

17. J. van Mieghem, "Hydrodynamic Instability," Compendium of Meteorology, American Meteorological Society, Boston, 1951, pp. 434-453. 